\title{
Invariance of Pontrjagin classes for Bott manifolds
}

\author{
SUYOUNG CHOI \\ MiKiYA MASUdA \\ SATOSHI MURAI
}

\begin{abstract}
A Bott manifold is the total space of some iterated $\mathbb{C P}^{1}$-bundles over a point. We prove that any graded ring isomorphism between the cohomology rings of two Bott manifolds preserves their Pontrjagin classes. Moreover, we prove that such an isomorphism is induced from a diffeomorphism if the Bott manifolds are $\mathbb{Z} / 2$ trivial, where a Bott manifold is called $\mathbb{Z} / 2$-trivial if its cohomology ring with $\mathbb{Z} / 2$-coefficients is isomorphic to that of a product of copies of $\mathbb{C P}^{1}$.
\end{abstract}

57R19, 57R20

\section{Introduction}

One of the fundamental problems in topology is to classify manifolds (up to diffeomorphism, homeomorphism etc) by invariants. Cohomology rings are not sufficient for this task in general. For example, surgery theory tells us that there are infinitely many diffeomorphism types in the family of closed smooth manifolds homotopy equivalent to a complex projective space $\mathbb{C P}^{n}$ when $n \geq 3$. However, surgery theory further tells us that they are distinguished by their Pontrjagin classes up to finite ambiguity, and this is true in general for the family of closed smooth manifolds homotopy equivalent to a fixed closed smooth manifold $X$ when $X$ is simply connected and of dimension at least 5 .

On the other hand, we have a feeling that most closed smooth manifolds do not admit an effective smooth $S^{1}$-action. For example, T Petrie [16] conjectures that if $M$ is a closed smooth manifold homotopy equivalent to $\mathbb{C P}^{n}$ and $M$ admits an effective smooth $S^{1}$-action, then a homotopy equivalence $f: M \rightarrow \mathbb{C P}^{n}$ preserves their Pontrjagin classes. Note that $\mathbb{C P}^{n}$ has an effective smooth action of $\left(S^{1}\right)^{n}$. The conjecture is not solved but many partial results in this direction are known. Among them, Petrie [17] showed that the conjecture is true if $M$ admits an effective smooth action of $\left(S^{1}\right)^{n}$. (See [11] for more details.)

A complete nonsingular toric variety (which we call a toric manifold) of complex dimension $n$ admits an effective algebraic action of $\left(\mathbb{C}^{*}\right)^{n}$, which is, in particular, 
an effective smooth action of $\left(S^{1}\right)^{n}$. The complex projective space $\mathbb{C P}^{n}$ is a typical example of a toric manifold. Motivated by Petrie's conjecture and his result mentioned above, the second-named author and D Y Suh [15] posed a problem that asks whether any cohomology ring isomorphism between toric manifolds preserves their Pontrjagin classes. (It is known that any cohomology ring isomorphism between toric manifolds preserves their Stiefel-Whitney classes [4, Appendix].) Little is known about this problem. One of our main purposes in this paper is to give an affirmative answer for a nice class of toric manifolds called Bott manifolds. We even show that any cohomology ring isomorphism is induced by a diffeomorphism for a certain subclass of Bott manifolds.

A Bott tower of height $n$ is a sequence of $\mathbb{C P}^{1}$-bundles

$$
B_{n} \stackrel{\pi_{n}}{\longrightarrow} B_{n-1} \stackrel{\pi_{n-1}}{\longrightarrow} \cdots \stackrel{\pi_{2}}{\longrightarrow} B_{1} \stackrel{\pi_{1}}{\longrightarrow} B_{0}=\{\text { a point }\},
$$

where $B_{j}$ is the projectivization $\mathbb{P}\left(\mathbb{C} \oplus L_{j}\right)$ of a trivial complex line bundle $\mathbb{C}$ and a complex line bundle $L_{j}$ over $B_{j-1}$, and $\pi_{j}: B_{j} \rightarrow B_{j-1}$ is the projection for $j=1,2, \ldots, n$. It is known that $B_{n}$ is a toric manifold and $B_{n}$ is called an $n$-stage Bott manifold or simply a Bott manifold.

If all the fibrations in (1-1) are trivial, then $B_{n}$ is diffeomorphic to $\left(\mathbb{C P}^{1}\right)^{n}$. The 1 -stage Bott manifold is $\mathbb{C P}^{1}$ and the 2 -stage Bott manifolds are Hirzebruch surfaces. As is well known, there are only two diffeomorphism types among Hirzebruch surfaces; $\left(\mathbb{C P}^{1}\right)^{2}$ and $\mathbb{C P}^{2} \# \overline{\mathbb{C P}^{2}}$, where $\overline{\mathbb{C P}^{2}}$ is $\mathbb{C P}^{2}$ with the opposite orientation. However, there are infinitely many diffeomorphism types among $n$-stage Bott manifolds when $n \geq 3$, and it is an interesting open question to classify them up to diffeomorphism or homeomorphism (Crowley and Kreck [9]).

Our first main result is the following.

Theorem 1.1 Any graded ring isomorphism between the cohomology rings with integer coefficients of two Bott manifolds preserves their Pontrjagin classes.

Since Bott manifolds are simply connected Kähler manifolds, by combining Theorem 1.1 with a result of Sullivan [18], we obtain the following corollary.

Corollary 1.2 The number of Bott manifolds whose cohomology ring is isomorphic to a given ring is finite up to diffeomorphism.

If a graded ring isomorphism between the cohomology rings of two smooth manifolds is induced by a diffeomorphism, then the isomorphism preserves their Pontrjagin classes. Hence, Theorem 1.1 provides evidence supporting the following conjecture. 
Strong cohomological rigidity conjecture for Bott manifolds Any graded ring isomorphism between the cohomology rings with integer coefficients of two Bott manifolds is induced by a diffeomorphism.

The statement of the conjecture above in particular implies that two Bott manifolds are diffeomorphic if their cohomology rings with integer coefficients are isomorphic as graded rings (in particular, if they are homotopy equivalent), and we call this weaker conjecture cohomological rigidity conjecture for Bott manifolds. No counterexamples are known to these conjectures and some partial results in this direction are known; for instance, the strong cohomological rigidity conjecture is true for the case of $\mathbb{Q}$ trivial Bott manifolds, where a Bott manifold $B_{n}$ is called $\mathbb{Q}$-trivial if $H^{*}\left(B_{n} ; \mathbb{Q}\right) \cong$ $\left.H^{*}\left((\mathbb{C P})^{1}\right)^{n} ; \mathbb{Q}\right)$ as graded rings (Choi and Masuda [3]). The strong cohomological conjecture also holds up to 3-stage Bott manifolds, and the cohomological rigidity conjecture holds for 4-stage Bott manifolds (Choi [2]).

We say that a Bott manifold $B_{n}$ is $\mathbb{Z} / 2$-trivial if $H^{*}\left(B_{n} ; \mathbb{Z} / 2\right) \cong H^{*}\left(\left(\mathbb{C P}^{1}\right)^{n} ; \mathbb{Z} / 2\right)$ as graded rings, where $\mathbb{Z} / 2:=\mathbb{Z} / 2 \mathbb{Z}$. We note that a Bott manifold is $\mathbb{Z} / 2$-trivial if and only if all line bundles $L_{j}$ in (1-1) are spin. Our second main result is this:

Theorem 1.3 The strong cohomological rigidity conjecture holds for $\mathbb{Z} / 2$-trivial Bott manifolds, namely, any graded ring isomorphism between the cohomology rings (with integer coefficients) of two $\mathbb{Z} / 2$-trivial Bott manifolds is induced by a diffeomorphism.

There are infinitely many diffeomorphism types among the $\mathbb{Z} / 2$-trivial $n$-stage Bott manifolds when $n \geq 3$, while there are only finitely many diffeomorphism types among the $\mathbb{Q}$-trivial $n$-stage Bott manifolds for any $n$. Therefore, the family of $\mathbb{Z} / 2$-trivial Bott manifolds is much larger than that of $\mathbb{Q}$-trivial Bott manifolds, but the former family does not contain the latter. For instance, a 2-stage Bott manifold $\mathbb{C P}^{2} \# \overline{\mathbb{C P}^{2}}$ is not $\mathbb{Z} / 2$-trivial but it is $\mathbb{Q}$-trivial. We remark that, for each $n$, only $\left(\mathbb{C P}^{1}\right)^{n}$ is a Bott manifold that is both $\mathbb{Z} / 2$-trivial and $\mathbb{Q}$-trivial.

The rigidity conjectures mentioned above may be posed more generally for toric manifolds or some related family of manifolds [15]. It is known that the strong cohomological rigidity conjecture does not hold for arbitrary toric manifolds, while no counterexamples are known to the cohomological rigidity problems for toric manifolds. A real analogue of the rigidity problems has also been studied. See the survey papers by Choi, Masuda and Suh [5; 15] for details.

This paper is organized as follows. In Section 2 we review some known facts about the cohomology rings of Bott manifolds. In Section 3 we introduce new bases for the cohomology rings and restate some facts mentioned in Section 2. In Section 4 we analyze graded ring isomorphisms between the cohomology rings of two Bott manifolds, 
where the new bases introduced in Section 3 play a role. We prove Theorem 1.1 in Section 5 and Theorem 1.3 in Section 6. In Section 7, we observe that the invariance of Pontrjagin classes under cohomology ring isomorphisms hold in a slightly wider class of manifolds than that of Bott manifolds. Finally, in Section 8 we make some remarks on automorphisms of the cohomology ring of a Bott manifold, which clarifies the difficulties for solving the strong cohomological rigidity conjecture for Bott manifolds completely.

\section{Cohomology rings of Bott manifolds}

In this section we will recall some known facts on the cohomology rings of Bott manifolds and the quotient construction of Bott manifolds.

We denote by $\alpha_{j}$ the first Chern class of the complex line bundle $L_{j}$ used to construct the Bott tower (1-1). It follows from the Borel-Hirzebruch formula [1] that $H^{*}\left(B_{j} ; \mathbb{Z}\right)$ is a free module over $H^{*}\left(B_{j-1} ; \mathbb{Z}\right)$ through the map $\pi_{j}^{*}: H^{*}\left(B_{j-1} ; \mathbb{Z}\right) \rightarrow H^{*}\left(B_{j} ; \mathbb{Z}\right)$ on two generators 1 and $x_{j}$, of degrees 0 and 2 respectively, where $x_{j}$ is the first Chern class of the tautological line bundle $\gamma_{j}$ over $B_{j}$, and that the ring structure is determined by the single relation

$$
x_{j}^{2}=\pi_{j}^{*}\left(\alpha_{j}\right) x_{j} .
$$

Using the formula inductively on $j$ and regarding $H^{*}\left(B_{j} ; \mathbb{Z}\right)$ as a subring of $H^{*}\left(B_{n} ; \mathbb{Z}\right)$ through the projections in (1-1), we see that

$$
H^{*}\left(B_{n} ; \mathbb{Z}\right)=\mathbb{Z}\left[x_{1}, \ldots, x_{n}\right] /\left(x_{j}^{2}-\alpha_{j} x_{j} \mid j=1,2, \ldots, n\right),
$$

where $\alpha_{1}=0$. The following lemma easily follows from (2-1).

Lemma 2.1 Let $k$ be a positive integer less than or equal to $n$. Then the set

$$
\left\{x_{i_{1}} x_{i_{2}} \cdots x_{i_{k}} \mid 1 \leq i_{1}<i_{2}<\cdots<i_{k} \leq n\right\}
$$

is an additive basis of $H^{2 k}\left(B_{n} ; \mathbb{Z}\right)$.

The Pontrjagin class of a Bott manifold has a simple expression as is shown in the following lemma.

Lemma 2.2 The Pontrjagin class $p\left(B_{n}\right)$ of the Bott manifold $B_{n}$ is given by

$$
p\left(B_{n}\right)=\prod_{j=1}^{n}\left(1+\left(2 x_{j}-\alpha_{j}\right)^{2}\right)=\prod_{j=1}^{n}\left(1+\alpha_{j}^{2}\right) \in H^{*}\left(B_{n} ; \mathbb{Z}\right),
$$

where $\left(2 x_{j}-\alpha_{j}\right)^{2}=\alpha_{j}^{2}$ because $x_{j}^{2}=\alpha_{j} x_{j}$. 
Proof This lemma is known, but since there seems to be no paper in the literature that mentions the formula explicitly, we shall give a proof.

Since $\pi_{j}: B_{j} \rightarrow B_{j-1}$ is the projectivization of the Whitney sum of the trivial line bundle $\mathbb{C}$ and the line bundle $L_{j}$ over $B_{j-1}$, the tangent bundle $T B_{j}$ of $B_{j}$ splits into

$$
T B_{j}=T_{f} B_{j} \oplus \pi_{j}^{*}\left(T B_{j-1}\right),
$$

where $T_{f} B_{j}$ denotes the complex line bundle along the fibers of the fiber bundle $\pi_{j}: B_{j} \rightarrow B_{j-1}$ and $\pi_{j}^{*}\left(T B_{j-1}\right)$ is the pullback of the tangent bundle $T B_{j-1}$ of the base space $B_{j-1}$ by the projection $\pi_{j}$. Since $x_{j}$ is the first Chern class of the tautological line bundle $\gamma_{j}$ over $B_{j}$ and the total Chern class of $\mathbb{C} \oplus L_{j}$ is $1+\alpha_{j}$, it follows from [1, (2), page 515] that the total Chern class of the complex line bundle $T_{f} B_{j}$ is given by $1-2 x_{j}+\alpha_{j}$ and hence its total Pontrjagin class is given by $1+\left(2 x_{j}-\alpha_{j}\right)^{2}$. This together with (2-2) shows that $p\left(B_{j}\right)=\left(1+\left(2 x_{j}-\alpha_{j}\right)^{2}\right) p\left(B_{j-1}\right)$ because $H^{*}\left(B_{n} ; \mathbb{Z}\right)$ has no 2 -torsion. Then the lemma follows by applying the above formula inductively on $j$.

For $u=\sum_{i=1}^{n} c_{i} x_{i} \in H^{2}\left(B_{n} ; \mathbb{Z}\right)$ with $c_{i} \in \mathbb{Z}$, we define

$$
\operatorname{ht}(u)=\max \left\{i \in[n]=\{1,2, \ldots, n\} \mid c_{i} \neq 0\right\}
$$

and call it the height of $u$. Note that $\operatorname{ht}\left(\alpha_{j}\right)<\operatorname{ht}\left(x_{j}\right)=j$. We say that a pair of primitive elements in $H^{2}\left(B_{n} ; \mathbb{Z}\right)$ is a primitive vanishing pair if the product of the elements vanishes. Note that a pair $\left(x_{j}, x_{j}-\alpha_{j}\right)$ is a primitive vanishing pair for any $j \in[n]$.

Lemma 2.3 [3, Lemma 2.2] A primitive vanishing pair is of the form

$$
\left(a x_{j}+u, a\left(x_{j}-\alpha_{j}\right)-u\right) \text { or }\left(a x_{j}+u,-a\left(x_{j}-\alpha_{j}\right)+u\right),
$$

where $a \in \mathbb{Z} \backslash\{0\}, u \in H^{2}\left(B_{n} ; \mathbb{Z}\right), u\left(u+a \alpha_{j}\right)=0$ and ht $(u)<j$.

Corollary 2.4 [3, Corollary 2.1] Primitive square-zero elements in $H^{2}\left(B_{n} ; \mathbb{Z}\right)$ are of the form $\pm\left(x_{j}-\frac{1}{2} \alpha_{j}\right)$ if $\alpha_{j} \equiv 0(\bmod 2)$ and $\pm\left(2 x_{j}-\alpha_{j}\right)$ otherwise.

Next we shall review the quotient construction of Bott manifolds [8, Proposition 3.1]. Remember that $\alpha_{1}=0$, and write

$$
\alpha_{j}=\sum_{i=1}^{j-1} A_{j}^{i} x_{i} \quad \text { for } j=2,3, \ldots, n
$$

with $A_{j}^{i} \in \mathbb{Z}$. Let $S^{2 d-1}$ be the unit sphere of $\mathbb{C}^{d}$ for $d=1,2$. Then the Bott manifold $B_{n}$ in (1-1) can be obtained as the quotient of $\left(S^{3}\right)^{n}$ by the free action 
of $\left(S^{1}\right)^{n}$ defined by

$$
\begin{aligned}
& \left(g_{1}, g_{2}, \ldots, g_{n}\right) \cdot\left(\left(z_{1}, w_{1}\right),\left(z_{2}, w_{2}\right), \ldots,\left(z_{n}, w_{n}\right)\right) \\
& \quad=\left(\left(g_{1} z_{1}, g_{1} w_{1}\right),\left(\left(g_{1}^{-A_{2}^{1}}\right) g_{2} z_{2}, g_{2} w_{2}\right), \ldots,\left(\left(\prod_{k=1}^{n-1} g_{k}^{-A_{n}^{k}}\right) g_{n} z_{n}, g_{n} w_{n}\right)\right),
\end{aligned}
$$

where $g_{i} \in S^{1}$ and $\left(z_{i}, w_{i}\right) \in S^{3}$ for $i=1,2, \ldots, n$. The projections

$$
\left(\left(z_{1}, w_{1}\right), \ldots,\left(z_{n}, w_{n}\right)\right) \rightarrow\left(\left(z_{1}, w_{1}\right), \ldots,\left(z_{n-1}, w_{n-1}\right)\right) \rightarrow \cdots \rightarrow\left(z_{1}, w_{1}\right)
$$

induce the Bott tower (1-1). The tautological line bundle $\gamma_{j}$ over $B_{j}$ can be described as the quotient of the trivial complex line bundle $\left(S^{3}\right)^{n} \times \mathbb{C} \rightarrow\left(S^{3}\right)^{n}$ by the action of $\left(S^{1}\right)^{n}$ on the total space $\left(S^{3}\right)^{n} \times \mathbb{C}$ defined as

$$
g \cdot((z, w), u)=\left(g \cdot(z, w), g_{j}^{-1} u\right) \quad(u \in \mathbb{C}),
$$

where $g=\left(g_{1}, g_{2}, \ldots, g_{n}\right),(z, w)=\left(\left(z_{1}, w_{1}\right),\left(z_{2}, w_{2}\right), \ldots,\left(z_{n}, w_{n}\right)\right)$ and $g \cdot(z, w)$ denotes the action defined in (2-4).

\section{Base change}

We set

$$
y_{j}=x_{j}-\frac{1}{2} \alpha_{j} \quad \text { for } j=1,2, \ldots, n
$$

Then we have

$$
4 y_{j}^{2}=\left(2 x_{j}-\alpha_{j}\right)^{2}=\alpha_{j}^{2} \quad \text { in } H^{*}\left(B_{n} ; \mathbb{Z}\right)
$$

and it follows from Lemma 2.2 that

$$
p\left(B_{n}\right)=\prod_{i=1}^{n}\left(1+4 y_{j}^{2}\right) .
$$

If $\alpha_{j} \equiv 0(\bmod 2)$, then $y_{j}$ is integral, that is, an element of $H^{2}\left(B_{n} ; \mathbb{Z}\right)$.

Lemma 3.1 If $\alpha_{i} \equiv \alpha_{j} \equiv 0(\bmod 2)$ and $y_{i} \equiv y_{j}(\bmod 2)$, then $i=j$.

Proof Since ht $\left(\alpha_{i}\right)<i$ and $\mathrm{ht}\left(\alpha_{j}\right)<j$, the assumption $y_{i} \equiv y_{j}(\bmod 2)$ implies $x_{i} \equiv x_{j}(\bmod 2)$ and hence $i=j$ because $\left\{x_{1}, x_{2}, \ldots, x_{n}\right\}$ is an additive basis of $H^{2}\left(B_{n} ; \mathbb{Z}\right)($ cf Lemma 2.1). 
Let $A_{j}^{i}(i<j)$ be the integers defined in (2-3). Setting $A_{j}^{i}=0$ for $i \geq j$, we obtain an integral strictly upper-triangular $n \times n$ matrix $A$ with $A_{j}^{i}$ as the $(i, j)^{\text {th }}$ entry. Then it follows from (3-1) that $\left(y_{1}, \ldots, y_{n}\right)=\left(x_{1}, \ldots, x_{n}\right)\left(E-\frac{1}{2} A\right)$, where $E$ is the identity matrix. Here $E-\frac{1}{2} A$ is an upper-triangular unipotent matrix and so is its inverse. Therefore, if we denote the $(i, j)^{\text {th }}$ entry of $\left(E-\frac{1}{2} A\right)^{-1}$ by $a_{j}^{i}$, then

$$
x_{j}=\sum_{i \leq j} a_{j}^{i} y_{i} \quad \text { and } \quad \frac{1}{2} \alpha_{j}=x_{j}-y_{j}=\sum_{i<j} a_{j}^{i} y_{i} .
$$

By (3-2) and (3-4), we have

$$
y_{j}^{2}=\left(\frac{\alpha_{j}}{2}\right)^{2}=\left(\sum_{i<j} a_{j}^{i} y_{i}\right)^{2} \quad \text { in } H^{*}\left(B_{n} ; \mathbb{Q}\right) .
$$

The following lemma easily follows from (3-5) or Lemma 2.1 .

Lemma 3.2 Let $k$ be a positive integer less than or equal to $n$. Then the set

$$
\left\{y_{i_{1}} y_{i_{2}} \cdots y_{i_{k}} \mid 1 \leq i_{1}<i_{2}<\cdots<i_{k} \leq n\right\}
$$

is an additive basis of $H^{2 k}\left(B_{n} ; \mathbb{Q}\right)$ over $\mathbb{Q}$.

\section{Cohomology ring isomorphisms}

Let $\mathcal{B}_{n}$ be the set of integral strictly upper-triangular $n \times n$ matrices. Since the Bott manifold $B_{n}$ in (1-1) is determined by a matrix $A \in \mathcal{B}_{n}$, we will denote $B_{n}$ by $M(A)$. We remark that it can happen that $M(A)$ and $M(B)$ are diffeomorphic even if $A, B \in \mathcal{B}_{n}$ are different.

Henceforth the cohomology elements $x_{j}, y_{j}, \alpha_{j}$ for $M(A)$ will be denoted by $x_{j}^{A}, y_{j}^{A}$, $\alpha_{j}^{A}$, respectively, to avoid confusion.

Proposition 4.1 Suppose that $\psi: H^{*}(M(A) ; \mathbb{Q}) \rightarrow H^{*}(M(B) ; \mathbb{Q})$ is a graded ring isomorphism. Then there are nonzero $q_{1}, \ldots, q_{n} \in \mathbb{Q}$ and a permutation $\sigma$ on $[n]$ such that

$$
\psi\left(y_{j}^{A}\right)=q_{j} y_{\sigma(j)}^{B} \quad \text { for } j=1, \ldots, n
$$

Proof We prove the proposition by induction on $j$. When $j=1$, we have $\psi\left(y_{1}^{A}\right)^{2}=0$ because $\left(y_{1}^{A}\right)^{2}=0$. Therefore $\psi\left(y_{1}^{A}\right)$ is a nonzero scalar multiple of $y_{\sigma(1)}^{B}$ for some $\sigma(1) \in[n]$ by Corollary 2.4. 
Suppose that

$$
\psi\left(y_{j}^{A}\right)=q_{j} y_{\sigma(j)}^{B} \quad \text { for } j<k .
$$

Then, since

$$
\left(q_{j} y_{\sigma(j)}^{B}\right)^{2}=\psi\left(y_{j}^{A}\right)^{2}=\psi\left(\left(y_{j}^{A}\right)^{2}\right)=\psi\left(\left(\sum_{i<j} a_{j}^{i} y_{i}^{A}\right)^{2}\right)=\left(\sum_{i<j} a_{j}^{i} q_{i} y_{\sigma(i)}^{B}\right)^{2}
$$

one can see by induction that for $j<k$,

$$
\left(y_{\sigma(j)}^{B}\right)^{2} \text { is a linear combination of } y_{\sigma\left(i_{1}\right)}^{B} y_{\sigma\left(i_{2}\right)}^{B} \text { with } i_{1}<i_{2}<j .
$$

Set $S=\{\sigma(1), \ldots, \sigma(k-1)\}$. Since $\psi$ is an isomorphism, it follows from (4-1) that $\psi\left(y_{k}^{A}\right)$ is not a linear combination of $y_{\sigma(1)}^{B}, \ldots, y_{\sigma(k-1)}^{B}$. Therefore, there is an $m \in[n] \backslash S$ such that

$$
\psi\left(y_{k}^{A}\right)=\sum_{s \leq m \text { or } s \in S} b_{s} y_{s}^{B} \quad\left(b_{s} \in \mathbb{Q} \text { and } b_{m} \neq 0\right) .
$$

Taking the square of both sides, it follows from (3-5) and (4-1) that

and hence

$$
\psi\left(y_{k}^{A}\right)^{2}=\psi\left(\left(y_{k}^{A}\right)^{2}\right)=\psi\left(\left(\sum_{i<k} a_{k}^{i} y_{i}^{A}\right)^{2}\right)=\left(\sum_{i<k} a_{k}^{i} q_{i} y_{\sigma(i)}^{B}\right)^{2}
$$

$$
\psi\left(y_{k}^{A}\right)^{2} \text { is a linear combination of } y_{\sigma\left(i_{1}\right)}^{B} y_{\sigma\left(i_{2}\right)}^{B} \text { with } i_{1}<i_{2}<k
$$

by (4-2). On the other hand, we claim that if $b_{t} \neq 0$ for some $t<m$ or $t \in S$ in the right-hand side of (4-3), then a nonzero scalar multiple of $y_{m}^{B} y_{t}^{B}$ appears in the square of the right-hand side of (4-3). Indeed, $\left(y_{s}^{B}\right)^{2}$ for $s \leq m$ is a linear combination of $y_{i}^{B} y_{j}^{B}$ with $i<j<m$ by (3-5) and $\left(y_{s}^{B}\right)^{2}$ for $s \in S$ is a linear combination of $y_{i}^{B} y_{j}^{B}$ with $i \neq j \in S$ by (4-2). Therefore the claim holds because $\left\{y_{i}^{B} y_{j}^{B} \mid 1 \leq i<j \leq n\right\}$ is an additive basis of $H^{4}(M(B) ; \mathbb{Q})$ by Lemma 3.2. However the claim contradicts (4-4) since $m \notin S$. Therefore, $\psi\left(y_{k}^{A}\right)=b_{m} y_{m}^{B}$. This completes the induction step and proves the proposition.

Lemma 4.2 Suppose that the graded ring isomorphism $\psi$ in Proposition 4.1 is induced by a graded ring isomorphism from $H^{*}(M(A) ; \mathbb{Z})$ to $H^{*}(M(B) ; \mathbb{Z})$. Then the rational number $q_{j}$ in Proposition 4.1 belongs to $\left\{ \pm \frac{1}{2}, \pm 1, \pm 2\right\}$ for $j=1,2, \ldots, n$, and

(1) $q_{j} \in\left\{ \pm \frac{1}{2}\right\}$ if and only if $\alpha_{j}^{A} \not \equiv 0(\bmod 2)$ and $\alpha_{\sigma(j)}^{B} \equiv 0(\bmod 2)$,

(2) $q_{j} \in\{ \pm 2\}$ if and only if $\alpha_{j}^{A} \equiv 0(\bmod 2)$ and $\alpha_{\sigma(j)}^{B} \not \equiv 0(\bmod 2)$.

Moreover, if $q_{j}= \pm 1$ for all $j$, then $\psi(p(M(A)))=p(M(B))$. 
Proof Since $\psi\left(x_{j}^{A}-\frac{1}{2} \alpha_{j}^{A}\right)=q_{j}\left(x_{\sigma(j)}^{B}-\frac{1}{2} \alpha_{\sigma(j)}^{B}\right)$ by Proposition 4.1, the former statement in the lemma follows from the fact that $\psi$ restricted to the cohomology rings with integer coefficients sends primitive elements to primitive elements and the latter follows from (3-3) and Proposition 4.1.

We fix a graded ring isomorphism $\psi: H^{*}(M(A) ; \mathbb{Z}) \rightarrow H^{*}(M(B) ; \mathbb{Z})$ until the end of Lemma 4.4. Since $\left(\psi\left(x_{j}^{A}\right), \psi\left(x_{j}^{A}-\alpha_{j}^{A}\right)\right)$ is a primitive vanishing pair, it follows from Lemma 2.3 that there are $a \in \mathbb{Z} \backslash\{0\}, u \in H^{2}(M(B) ; \mathbb{Z})$ and $k \in[n]$ such that

$$
\left(\psi\left(x_{j}^{A}\right), \psi\left(x_{j}^{A}-\alpha_{j}^{A}\right)\right)=\left\{\begin{array}{l}
\left(a x_{k}^{B}+u, a\left(x_{k}^{B}-\alpha_{k}^{B}\right)-u\right) \text { or } \\
\left(a x_{k}^{B}+u,-a\left(x_{k}^{B}-\alpha_{k}^{B}\right)+u\right),
\end{array}\right.
$$

where $u\left(u+a \alpha_{k}^{B}\right)=0$ and ht $(u)<k$. Remember that $a_{j}^{i}$ is the $(i, j)^{\text {th }}$ entry of the matrix $\left(E-\frac{1}{2} A\right)^{-1}$; in other words, $a_{j}^{i}$ is the rational number determined by (3-4).

With this understood, we have:

Lemma 4.3 In the former case of (4-5), $q_{j}=a$ (hence $q_{j}$ is an integer), $k=\sigma(j)$ and $a_{j}^{i}=0$ for $\sigma(i)>\sigma(j)$. In the latter case of (4-5), $\alpha_{j}^{A}=2 a q_{i}^{-1} y_{i}^{A}$ for some $i<j$.

Proof Since $2 y_{j}^{A}=x_{j}^{A}+\left(x_{j}^{A}-\alpha_{j}^{A}\right)$ and $\alpha_{j}^{A}=x_{j}^{A}-\left(x_{j}^{A}-\alpha_{j}^{A}\right)$, a simple computation using (4-5) shows that

$$
\left(\psi\left(2 y_{j}^{A}\right), \psi\left(\alpha_{j}^{A}\right)\right)= \begin{cases}\left(2 a y_{k}^{B}, 2 u+a \alpha_{k}^{B}\right) & \text { in the former case of (4-5), } \\ \left(2 u+a \alpha_{k}^{B}, 2 a y_{k}^{B}\right) & \text { in the latter case of (4-5). }\end{cases}
$$

In the former case of (4-5), we have $\psi\left(y_{j}^{A}\right)=a y_{k}^{B}$ by (4-6). This together with Proposition 4.1 shows that $q_{j}=a$ and $k=\sigma(j)$. Moreover, since $\psi\left(\alpha_{j}^{A}\right)=2 u+a \alpha_{k}^{B}$ by (4-6) and $h t(u)<k$, we have

$$
\operatorname{ht}\left(\psi\left(\alpha_{j}^{A}\right)\right)<k=\sigma(j)
$$

while since $\alpha_{j}^{A}=2 \sum_{i<j} a_{j}^{i} y_{i}^{A}$ by (3-4), we have

$$
\psi\left(\alpha_{j}^{A}\right)=2 \sum_{i<j} a_{j}^{i} q_{i} y_{\sigma(i)}^{B}
$$

by Proposition 4.1. These show that $a_{j}^{i}=0$ for $\sigma(i)>\sigma(j)$.

In the latter case of (4-5), $\psi\left(\alpha_{j}^{A}\right)=2 a y_{k}^{B}$ by (4-6). Therefore $\alpha_{j}^{A}=2 a q_{i}^{-1} y_{i}^{A}$ for some $i \in[n]$ by Proposition 4.1 and $j>\operatorname{ht}\left(\alpha_{j}^{A}\right)=\operatorname{ht}\left(y_{i}^{A}\right)=i$. 
Note that $2 a q_{i}^{-1}$ in Lemma 4.3 is a nonzero integer because $a \in \mathbb{Z} \backslash\{0\}$ and $q_{i} \in$ $\left\{ \pm \frac{1}{2}, \pm 1, \pm 2\right\}$ by Lemma 4.2. We say that $\alpha_{j}$ is of exceptional type if $\alpha_{j}=c y_{i}$ for some nonzero integer $c$ and $i<j$ and is of even exceptional type if the nonzero integer $c$ is even.

Lemma 4.4 Suppose $q_{j}= \pm \frac{1}{2}$. Then there are $i<j, c \in \mathbb{Z} \backslash\{0\}$, such that $\alpha_{j}^{A}=c y_{i}^{A}$. If $\alpha_{j}^{A}$ is not of even exceptional type, then $c$ is odd and $q_{i}= \pm 2$. If neither $\alpha_{j}^{A}$ nor $\alpha_{\sigma(i)}^{B}$ are of even exceptional type, then $\alpha_{\sigma(i)}^{B}=d y_{\sigma(j)}^{B}$ with some odd integer $d$.

Proof Since $q_{j}= \pm \frac{1}{2}$ by assumption, it is not an integer. Lemma 4.3 then says that the latter case of (4-5) must occur and $\alpha_{j}^{A}=2 a q_{i}^{-1} y_{i}^{A}$. Setting $c=2 a q_{i}^{-1}$, we have

$$
\alpha_{j}^{A}=c y_{i}^{A}
$$

where $c$ is a nonzero integer. If $\alpha_{j}^{A}$ is not of even exceptional type, then $c=2 a q_{i}^{-1}$ is odd and hence $q_{i}$ must be \pm 2 (and $a$ is odd).

It remains to prove the last assertion in the lemma. Suppose that neither $\alpha_{j}^{A}$ nor $\alpha_{\sigma(i)}^{B}$ are of even exceptional type. Then $q_{i}= \pm 2$ as observed above, and since $\psi\left(y_{i}^{A}\right)=q_{i} y_{\sigma(i)}^{B}$ by Proposition 4.1, we have $\psi^{-1}\left(y_{\sigma(i)}^{B}\right)= \pm \frac{1}{2} y_{i}^{A}$. Then the same argument above applied to $\psi^{-1}\left(y_{\sigma(i)}^{B}\right)= \pm \frac{1}{2} y_{i}^{A}$ tells us that there are $\ell \in[n]$ and $d \in \mathbb{Z} \backslash\{0\}$ such that

$$
\alpha_{\sigma(i)}^{B}=d y_{\ell}^{B} .
$$

Here $d$ is odd because $\alpha_{\sigma(i)}^{B}$ is not of even exceptional type by assumption. In the sequel, it suffices to prove $\ell=\sigma(j)$.

Since $d$ is odd, it follows from (4-8) that $\alpha_{\ell}^{B} \equiv 0(\bmod 2)$ while $\alpha_{\sigma(j)}^{B} \equiv 0(\bmod 2)$ by Lemma 4.2 since $q_{j}= \pm \frac{1}{2}$. Thus it suffices to show $y_{\ell}^{B} \equiv y_{\sigma(j)}^{B}(\bmod 2)$ by Lemma 3.1, which we shall now prove. Since

$$
\pm y_{\sigma(j)}^{B}=\psi\left(2 y_{j}^{A}\right)=\psi\left(2 x_{j}^{A}-\alpha_{j}^{A}\right)=2 \psi\left(x_{j}^{A}\right)-\psi\left(\alpha_{j}^{A}\right),
$$

we have

$$
y_{\sigma(j)}^{B} \equiv \psi\left(\alpha_{j}^{A}\right)(\bmod 2) .
$$

Similarly, since $\pm \psi\left(y_{i}^{A}\right)=2 y_{\sigma(i)}^{B}=2 x_{\sigma(i)}^{B}-\alpha_{\sigma(i)}^{B}$, we have

$$
\psi\left(y_{i}^{A}\right) \equiv \alpha_{\sigma(i)}^{B}(\bmod 2) .
$$

Thus, since $c$ and $d$ are both odd integers, it follows from (4-9), (4-7), (4-10) and (4-8) that

$$
y_{\sigma(j)}^{B} \equiv \psi\left(\alpha_{j}^{A}\right) \equiv \psi\left(y_{i}^{A}\right) \equiv \alpha_{\sigma(i)}^{B} \equiv y_{\ell}^{B}(\bmod 2),
$$

proving the desired congruence relation. 
Lemma 4.5 For $A \in \mathcal{B}_{n}$, there is $B \in \mathcal{B}_{n}$ such that none of $\alpha_{1}^{B}, \ldots, \alpha_{n}^{B}$ are of even exceptional type and there is a graded ring isomorphism $\psi: H^{*}(M(A) ; \mathbb{Z}) \rightarrow$ $H^{*}(M(B) ; \mathbb{Z})$ such that $\psi(p(M(A)))=p(M(B))$.

Proof Suppose that $\alpha_{j}^{A}$ is of even exceptional type but $\alpha_{k}^{A}$ for $k<j$ is not. In the following we will find $B \in \mathcal{B}_{n}$ such that $\alpha_{k}^{B}$ for $k<j$ is not of even exceptional type but ht $\left(\alpha_{j}^{B}\right)<\mathrm{ht}\left(\alpha_{j}^{A}\right)$ and that $H^{*}(M(A) ; \mathbb{Z})$ and $H^{*}(M(B) ; \mathbb{Z})$ are isomorphic as graded rings. If $\alpha_{j}^{B}$ is still of even exceptional type, then we repeat the argument until we reach $B$ such that $\alpha_{k}^{B}$ for $k \leq j$ is not of even exceptional type. Note that this can be achieved because if $\operatorname{ht}\left(\alpha_{j}^{B}\right)=0$, then $\alpha_{j}^{B}=0$, which is not of even exceptional type. Doing this procedure inductively on $j$, we finally reach the desired $B$ in the lemma.

Since $\alpha_{j}^{A}$ is of even exceptional type by assumption, we have

$$
\alpha_{j}^{A}=c\left(x_{i}^{A}-\frac{1}{2} \alpha_{i}^{A}\right)
$$

with some nonzero even integer $c$ and $i<j$. We define a matrix $B$ of size $n$ by

$$
B_{k}^{\ell}= \begin{cases}A_{k}^{\ell} & \text { if } k \neq j \text { and } \ell \neq i \\ A_{k}^{i}+\frac{c}{2} A_{k}^{j} & \text { if } k \neq j \text { and } \ell=i \\ -\frac{c}{2} A_{i}^{\ell} & \text { if } k=j\end{cases}
$$

Since $A \in \mathcal{B}_{n}$ and $i<j$, the matrix $B$ is indeed in $\mathcal{B}_{n}$ (ie, $B_{k}^{\ell}=0$ for $\ell \geq k$ ), and

$$
\alpha_{k}^{B}= \begin{cases}\sum_{\ell=1}^{n} A_{k}^{\ell} x_{\ell}^{B}+\frac{c}{2} A_{k}^{j} x_{i}^{B} & \text { if } k \neq j, \\ -\frac{c}{2} \sum_{\ell=1}^{n} A_{i}^{\ell} x_{\ell}^{B} & \text { if } k=j .\end{cases}
$$

Note that since $i<j, A_{i}^{j}=0$ and hence it follows from (4-12) that

$$
\alpha_{j}^{B}=-\frac{c}{2} \alpha_{i}^{B} .
$$

We define

$$
\psi\left(x_{\ell}^{A}\right)= \begin{cases}x_{\ell}^{B} & \text { if } \ell \neq j, \\ x_{j}^{B}+\frac{c}{2} x_{i}^{B} & \text { if } \ell=j .\end{cases}
$$

This clearly induces an isomorphism $\psi: \mathbb{Z}\left[x_{1}^{A}, \ldots, x_{n}^{A}\right] \rightarrow \mathbb{Z}\left[x_{1}^{B}, \ldots, x_{n}^{B}\right]$ between polynomial rings. We claim that $\psi$ induces a graded isomorphism from $H^{*}(M(A) ; \mathbb{Z})$ to $H^{*}(M(B) ; \mathbb{Z})$. Indeed, when $k \neq j$, it follows from (4-14) and (4-12) that

and hence

$$
\psi\left(\alpha_{k}^{A}\right)=\psi\left(\sum_{\ell=1}^{n} A_{k}^{\ell} x_{\ell}^{A}\right)=\sum_{\ell=1}^{n} A_{k}^{\ell} x_{\ell}^{B}+\frac{c}{2} A_{k}^{j} x_{i}^{B}=\alpha_{k}^{B}
$$

$$
\psi\left(x_{k}^{A}\left(x_{k}^{A}-\alpha_{k}^{A}\right)\right)=x_{k}^{B}\left(x_{k}^{B}-\alpha_{k}^{B}\right) \quad \text { when } k \neq j
$$


When $k=j$, we have

$$
\begin{aligned}
\psi\left(x_{j}^{A}\left(x_{j}^{A}-\alpha_{j}^{A}\right)\right) & =\psi\left(x_{j}^{A}\left(x_{j}^{A}-c\left(x_{i}^{A}-\frac{1}{2} \alpha_{i}^{A}\right)\right)\right) \quad \text { by (4-11) } \\
& =\left(x_{j}^{B}+\frac{c}{2} x_{i}^{B}\right)\left(x_{j}^{B}-\frac{c}{2} x_{i}^{B}+\frac{c}{2} \alpha_{i}^{B}\right) \quad \text { by (4-14), (4-15) } \\
& =x_{j}^{B}\left(x_{j}^{B}+\frac{c}{2} \alpha_{i}^{B}\right)-\frac{c^{2}}{4} x_{i}^{B}\left(x_{i}^{B}-\alpha_{i}^{B}\right) \\
& =x_{j}^{B}\left(x_{j}^{B}-\alpha_{j}^{B}\right)-\frac{c^{2}}{4} x_{i}^{B}\left(x_{i}^{B}-\alpha_{i}^{B}\right) \quad \text { by (4-13). }
\end{aligned}
$$

(4-16) and (4-17) together with (2-1) show that $\psi$ induces a graded ring isomorphism from $H^{*}(M(A) ; \mathbb{Z})$ to $H^{*}(M(B) ; \mathbb{Z})$.

Since $\operatorname{ht}\left(\alpha_{j}^{A}\right)=i$ by (4-11) and $\operatorname{ht}\left(\alpha_{j}^{B}\right)=\operatorname{ht}\left(\alpha_{i}^{B}\right)<i$ by (4-13), we have $\operatorname{ht}\left(\alpha_{j}^{B}\right)<$ $\operatorname{ht}\left(\alpha_{j}^{A}\right)$. Moreover, the isomorphism $\psi$ defined in (4-14) is represented as a unipotent upper-triangular matrix with respect to the basis $x_{1}^{A}, \ldots, x_{n}^{A}$ of $H^{2}(M(A) ; \mathbb{Z})$ and $x_{1}^{B}, \ldots, x_{n}^{B}$ of $H^{2}(M(B) ; \mathbb{Z})$, so $q_{j}=1$ for any $j$ and hence $\psi(p(M(A)))=$ $p(M(B))$ by Lemma 4.2.

Remark 4.6 The graded ring isomorphism $\psi$ in the proof above is actually induced by a diffeomorphism. This follows from Theorem 6.2 mentioned in Section 6. One can also see it when using the quotient construction of Bott manifolds.

\section{Proof of Theorem 1.1}

This section is devoted to the proof of Theorem 1.1 in the introduction.

Let $\psi: H^{*}(M(A) ; \mathbb{Z}) \rightarrow H^{*}(M(B) ; \mathbb{Z})$ be a graded ring isomorphism. By (3-3), what we must prove is

$$
\psi\left(\prod_{j=1}^{n}\left(1+4\left(y_{j}^{A}\right)^{2}\right)\right)=\prod_{j=1}^{n}\left(1+4\left(y_{j}^{B}\right)^{2}\right) \in H^{*}(M(B) ; \mathbb{Z}) .
$$

We may assume that none of $\alpha_{1}^{A}, \ldots, \alpha_{n}^{A}$ and $\alpha_{1}^{B}, \ldots, \alpha_{n}^{B}$ are of even exceptional type by Lemma 4.5 . Since

$$
\psi\left(y_{j}^{A}\right)=q_{j} y_{\sigma(j)}^{B}
$$

by Proposition 4.1, $\psi\left(1+4\left(y_{j}^{A}\right)^{2}\right)=1+4\left(y_{\sigma(j)}^{B}\right)^{2}$ if $q_{j}= \pm 1$. Therefore, we shall treat the case where $q_{j}= \pm \frac{1}{2}$ or \pm 2 by Lemma 4.2 .

Suppose $q_{j}= \pm \frac{1}{2}$. Then there is $i<j$ such that

$$
\alpha_{j}^{A}=c y_{i}^{A} \quad \text { and } \quad \alpha_{\sigma(i)}^{B}=d y_{\sigma(j)}^{B}
$$


with some odd integers $c, d$ and $q_{i}= \pm 2$ by Lemma 4.4. We shall show that

$$
\psi\left(\left(1+4\left(y_{j}^{A}\right)^{2}\right)\left(1+4\left(y_{i}^{A}\right)^{2}\right)\right)=\left(1+4\left(y_{\sigma(j)}^{B}\right)^{2}\right)\left(1+4\left(y_{\sigma(i)}^{B}\right)^{2}\right) .
$$

It follows from (3-2) and (5-1) that

$$
4\left(y_{j}^{A}\right)^{2}=\left(\alpha_{j}^{A}\right)^{2}=c^{2}\left(y_{i}^{A}\right)^{2}
$$

and applying $\psi$ to the first and last elements in the identity above, we obtain

$$
\left(y_{\sigma(j)}^{B}\right)^{2}=4 c^{2}\left(y_{\sigma(i)}^{B}\right)^{2}
$$

since $q_{j}= \pm \frac{1}{2}$ and $q_{i}= \pm 2$. Here $4\left(y_{\sigma(i)}^{B}\right)^{2}=\left(\alpha_{\sigma(i)}^{B}\right)^{2}$ by (3-2) and $\left(\alpha_{\sigma(i)}^{B}\right)^{2}=$ $d^{2}\left(y_{\sigma(j)}^{B}\right)^{2}$ by (5-1). Therefore, (5-4) turns into

$$
\left(y_{\sigma(j)}^{B}\right)^{2}=c^{2} d^{2}\left(y_{\sigma(j)}^{B}\right)^{2} .
$$

When $\left(y_{\sigma(i)}^{B}\right)^{2} \neq 0,(5-5)$ implies $c^{2} d^{2}=1$ and hence $c^{2}=d^{2}=1$ because $c, d$ are integers. It follows from (5-3) and (5-4) that

$$
4\left(y_{j}^{A}\right)^{2}=\left(y_{i}^{A}\right)^{2} \quad \text { and } \quad\left(y_{\sigma(j)}^{B}\right)^{2}=4\left(y_{\sigma(i)}^{B}\right)^{2} .
$$

Therefore:

$$
\text { the left-hand side of } \begin{aligned}
(5-2) & =\psi\left(\left(1+\left(y_{i}^{A}\right)^{2}\right)\left(1+16\left(y_{j}^{A}\right)^{2}\right)\right. \\
& =\left(1+q_{i}^{2}\left(y_{\sigma(i)}^{B}\right)^{2}\right)\left(1+16 q_{j}^{2}\left(y_{\sigma(j)}^{B}\right)^{2}\right) \\
& =\text { the right-hand side of }(5-2)
\end{aligned}
$$

because $q_{j}= \pm \frac{1}{2}$ and $q_{i}= \pm 2$. When $\left(y_{\sigma(i)}^{B}\right)^{2}=0$, we have $\left(y_{\sigma(j)}^{B}\right)^{2}=0$ by (5-4) and $\left(y_{j}^{A}\right)^{2}=\left(y_{i}^{A}\right)^{2}=0$ because

$$
\psi\left(y_{j}^{A}\right)=y_{\sigma(j)}^{B} \quad \text { and } \quad \psi\left(y_{i}^{A}\right)=y_{\sigma(i)}^{B}
$$

and $\psi$ is an isomorphism. Therefore, (5-2) holds even when $\left(y_{\sigma(i)}^{B}\right)^{2}=0$.

When $q_{j}= \pm 2, \psi^{-1}\left(y_{\sigma(j)}^{B}\right)= \pm \frac{1}{2} y_{j}^{A}$. Therefore, the same argument as above applied for $\psi^{-1}$ shows that (5-2) also holds when $q_{j}= \pm 2$. This completes the proof of the theorem.

\section{Proof of Theorem 1.3}

The purpose of this section is to prove Theorem 1.3 in the introduction. We begin with the following lemma. 
Lemma 6.1 Let $A \in \mathcal{B}_{n}, \sigma$ a permutation on $[n]$ and let $P$ be the permutation matrix of $\sigma^{-1}$, that is, the $(i, j)^{\text {th }}$ entry of $P$ is 1 if $i=\sigma(j)$ and 0 otherwise. If $P A P^{-1} \in \mathcal{B}_{n}$, then there is a graded ring isomorphism $\psi_{\sigma}: H^{*}(M(A) ; \mathbb{Z}) \rightarrow H^{*}\left(M\left(P A P^{-1}\right) ; \mathbb{Z}\right)$ sending $x_{j}^{A}$ to $x_{\sigma(j)}^{P A P^{-1}}$ for $j=1,2, \ldots, n$ and it is induced by a diffeomorphism.

Proof Remember the quotient construction of Bott manifolds explained in Section 2. Let $\varphi_{\sigma}:\left(S^{3}\right)^{n} \rightarrow\left(S^{3}\right)^{n}$ be the coordinate change defined by

$$
\begin{aligned}
\varphi_{\sigma}\left(\left(z_{1}, w_{1}\right),\left(z_{2}, w_{2}\right), \ldots,\left(z_{n}, w_{n}\right)\right) & \\
& =\left(\left(z_{\sigma(1)}, w_{\sigma(1)}\right),\left(z_{\sigma(2)}, w_{\sigma(2)}\right), \ldots,\left(z_{\sigma(n)}, w_{\sigma(n)}\right)\right)
\end{aligned}
$$

where we consider the $\left(S^{1}\right)^{n}$-action associated to the matrix $P A P^{-1}$ on the source space and the one associated to $A$ on the target space, and let $\phi_{\sigma}$ be the group automorphism of $\left(S^{1}\right)^{n}$ defined by $\phi_{\sigma}\left(g_{1}, g_{2}, \ldots, g_{n}\right)=\left(g_{\sigma(1)}, g_{\sigma(2)}, \ldots, g_{\sigma(n)}\right)$. Then $\varphi_{\sigma}$ is $\phi_{\sigma}$-equivariant, ie

$$
\varphi_{\sigma}(g \cdot(z, w))=\phi_{\sigma}(g) \cdot \varphi_{\sigma}((z, w))
$$

where $g=\left(g_{1}, \ldots, g_{n}\right)$ and $(z, w)=\left(\left(z_{1}, w_{1}\right),\left(z_{2}, w_{2}\right), \ldots,\left(z_{n}, w_{n}\right)\right)$. Indeed, the $j^{\text {th }}$ component of the left-hand side of (6-1) is

$$
\left(\left(\prod_{k=1}^{\sigma(j)-1} g_{k}^{-\left(P A P^{-1}\right)_{\sigma(j)}^{k}}\right) g_{\sigma(j)} z_{\sigma(j)}, g_{\sigma(j)} w_{\sigma(j)}\right)
$$

while that of the right-hand side of (6-1) is

$$
\left(\left(\prod_{i=1}^{j-1} g_{\sigma(i)}^{-A_{j}^{i}}\right) g_{\sigma(j)} z_{\sigma(j)}, g_{\sigma(j)} w_{\sigma(j)}\right)
$$

Here, since $P$ is the permutation matrix of $\sigma^{-1},\left(P A P^{-1}\right)_{\sigma(j)}^{\sigma(i)}=A_{j}^{i}$ and it is zero for $\sigma(i) \geq \sigma(j)$ or $i \geq j$ since both $P A P^{-1}$ and $A$ belong to $\mathcal{B}_{n}$; so we have

$$
\begin{aligned}
\prod_{k=1}^{\sigma(j)-1} g_{k}^{-\left(P A P^{-1}\right)_{\sigma(j)}^{k}}=\prod_{k=1}^{n} g_{k}^{-\left(P A P^{-1}\right)_{\sigma(j)}^{k}} & =\prod_{i=1}^{n} g_{\sigma(i)}^{-\left(P A P^{-1}\right)_{\sigma(j)}^{\sigma(i)}} \\
& =\prod_{i=1}^{n} g_{\sigma(i)}^{-A_{j}^{i}}=\prod_{i=1}^{j-1} g_{\sigma(i)}^{-A_{j}^{i}}
\end{aligned}
$$

This shows that (6-2) and (6-3) agree, which means that $\varphi_{\sigma}$ is $\phi_{\sigma}$-equivariant and hence $\varphi_{\sigma}$ induces a diffeomorphism from $M\left(P A P^{-1}\right)$ to $M(A)$. 
It remains to prove that the cohomology ring isomorphism induced by $\varphi_{\sigma}$ maps $x_{j}^{A}$ to $x_{\sigma(j)}^{P A P^{-1}}$. Remember that $x_{j}^{A}$ and $x_{\sigma(j)}^{P A P^{-1}}$ are the first Chern classes of the complex line bundles

$$
\gamma_{j}^{A} \text { and } \gamma_{\sigma(j)}^{P A P^{-1}}
$$

mentioned in Section 2, where the matrices $A$ and $P A P^{-1}$ are specified to avoid confusion. Therefore, it suffices to find a bundle isomorphism from $\gamma_{\sigma(j)}^{P A P^{-1}}$ to $\gamma_{j}^{A}$, which covers $\varphi_{\sigma}$. The map $f_{\sigma}$ from $\left(S^{3}\right)^{n} \times \mathbb{C}$ to itself defined by

$$
f_{\sigma}((z, w), u)=\left(\varphi_{\sigma}(z, w), u\right)
$$

satisfies $f_{\sigma}\left(g \cdot(z, w), g_{\sigma(j)}^{-1} u\right)=\left(\phi_{\sigma}(g) \cdot(z, w), g_{\sigma(j)}^{-1} u\right)$. Since $g_{\sigma(j)}$ is the $j^{\text {th }}$ component of $\phi_{\sigma}(g), f_{\sigma}$ induces the desired bundle isomorphism from $\gamma_{\sigma(j)}^{P A} P^{-1}$ to $\gamma_{j}^{A}$.

The following theorem due to $\mathrm{H}$ Ishida plays a role in our argument.

Theorem 6.2 [10] Let $A, B \in \mathcal{B}_{n}$ and $\psi: H^{*}(M(A) ; \mathbb{Z}) \rightarrow H^{*}(M(B) ; \mathbb{Z})$ be a graded ring isomorphism. If $\psi$ restricted to the degree-two cohomology groups is represented as an upper-triangular matrix with respect to the bases $x_{1}^{A}, \ldots, x_{n}^{A}$ of $H^{2}(M(A) ; \mathbb{Z})$ and $x_{1}^{B}, \ldots, x_{n}^{B}$ of $H^{2}(M(B) ; \mathbb{Z})$ defined in Section 2 , then $\psi$ is induced by a diffeomorphism.

Let $\psi: H^{*}(M(A) ; \mathbb{Z}) \rightarrow H^{*}(M(B) ; \mathbb{Z})$ be a graded ring isomorphism. By Proposition 4.1, there is a permutation $\sigma$ on $[n]$ such that $\psi\left(y_{j}^{A}\right)=q_{j} y_{\sigma(j)}^{B}$ with some nonzero $q_{j} \in \mathbb{Q}$ for any $j$.

Proposition 6.3 If $q_{j} \in\{ \pm 1\}$ for all $j$, then the isomorphism $\psi$ above is induced by a diffeomorphism.

Proof We may assume that none of $\alpha_{1}^{A}, \ldots, \alpha_{n}^{A}$ are of even exceptional type by Lemma 4.5 and Remark 4.6 (replacing $A$ with the matrix given in Lemma 4.5 if necessary). Let $a_{j}^{i}$ be the $(i, j)^{\text {th }}$ entry of $\left(E-\frac{1}{2} A\right)^{-1}$. Because none of $\alpha_{1}^{A}, \ldots, \alpha_{n}^{A}$ are of even exceptional type and $q_{k} \in\{ \pm 1\}$ for all $k$, we have $a_{j}^{i}=0$ for $\sigma(i)>$ $\sigma(j)$ by Lemma 4.3. This means that if $P$ is the permutation matrix of $\sigma^{-1}$, then $P\left(E-\frac{1}{2} A\right)^{-1} P^{-1}$ is a unipotent upper-triangular matrix because the $(\sigma(i), \sigma(j))^{\text {th }}$ entry of $P\left(E-\frac{1}{2} A\right)^{-1} P^{-1}$ is equal to the $(i, j)^{\text {th }}$ entry $a_{j}^{i}$ of $\left(E-\frac{1}{2} A\right)^{-1}$. Then, since $P\left(E-\frac{1}{2} A\right) P^{-1}=\left(P\left(E-\frac{1}{2} A\right)^{-1} P^{-1}\right)^{-1}$ is a unipotent upper-triangular matrix, $P A P^{-1}$ is a strictly upper-triangular matrix and hence $P A P^{-1} \in \mathcal{B}_{n}$. 
Let $\psi_{\sigma}: H^{*}(M(A) ; \mathbb{Z}) \rightarrow H^{*}\left(M\left(P A P^{-1}\right) ; \mathbb{Z}\right)$ be the graded ring isomorphism in Lemma 6.1. Then the graded ring isomorphism arising from the composition

$$
\psi \circ \psi_{\sigma}^{-1}: H^{*}\left(M\left(P A P^{-1}\right) ; \mathbb{Z}\right) \rightarrow H^{*}(M(A) ; \mathbb{Z}) \rightarrow H^{*}(M(B) ; \mathbb{Z})
$$

satisfies $\left(\psi \circ \psi_{\sigma}^{-1}\right)\left(y_{j}^{P A P^{-1}}\right)=q_{\sigma^{-1}(j)} y_{j}^{B}$ for any $j$ since $\psi_{\sigma}\left(x_{i}^{A}\right)=x_{\sigma(i)}^{P A P^{-1}}$ for any $i$. This means that $\psi \circ \psi_{\sigma}^{-1}$ satisfies the assumption in Theorem 6.2 and hence is induced by a diffeomorphism. Moreover, $\psi_{\sigma}$ is induced by a diffeomorphism by Lemma 6.1. This shows that $\psi$ is induced by a diffeomorphism.

Remember that $M(O)=\left(\mathbb{C P}^{1}\right)^{n}$ for the zero matrix $O \in \mathcal{B}_{n}$. We say that a Bott manifold $M(A)$ for $A \in \mathcal{B}_{n}$ is $\mathbb{Z} / 2-$ trivial if $H^{*}(M(A) ; \mathbb{Z} / 2) \cong H^{*}(M(O) ; \mathbb{Z} / 2)$ as graded rings.

Lemma 6.4 Let $A \in \mathcal{B}_{n}$. Then the following three statements are equivalent.

(1) $M(A)$ is $\mathbb{Z} / 2-$ trivial.

(2) $\alpha_{j}^{A} \equiv 0(\bmod 2)$ for $j=1,2, \ldots, n$.

(3) $A \equiv O(\bmod 2)$, ie every entry of $A$ is an even integer.

Proof The equivalence (2) $\Leftrightarrow(3)$ and the implication $(2) \Rightarrow(1)$ are obvious, so it suffices to prove the implication $(1) \Rightarrow(2)$. Suppose that $M(A)$ is $\mathbb{Z} / 2$-trivial. Then the square of any element in $H^{2}(M(A) ; \mathbb{Z} / 2)$ vanishes. Therefore $\alpha_{j}^{A} x_{j}^{A}\left(=x_{j}^{A^{2}}\right)$ vanishes in $H^{4}(M(A) ; \mathbb{Z} / 2)$ for any $j$. On the other hand, the set $\left\{x_{i}^{A} x_{j}^{A} \mid 1 \leq i<j \leq n\right\}$ is an additive basis of $H^{4}(M(A) ; \mathbb{Z} / 2)$ because it is an additive basis of $H^{4}(M(A) ; \mathbb{Z})$ by Lemma 2.1. Since $\alpha_{j}^{A}$ is a linear combination of $x_{i}^{A}$ for $1 \leq i<j$, this implies that $\alpha_{j}^{A}=0$ in $H^{2}(M(A) ; \mathbb{Z} / 2)$ for any $j$, proving (2).

We remark that $\alpha_{j}^{A}=c_{1}\left(L_{j}\right)$ where $L_{j}$ is the complex line bundle used to construct $M(A)$. Hence, the statement (2) in the above lemma is equivalent to the vanishing of the second Stiefel-Whitney class of $L_{j}$ for all $j=1,2, \ldots, n$. Therefore, a Bott manifold $M(A)$ is $\mathbb{Z} / 2$-trivial if and only if all complex line bundles $L_{j}$ used to construct $M(A)$ are spin.

Now we are in a position to prove our second main result stated in the introduction.

Proof of Theorem 1.3 Let $M(A)$ and $M(B)$ be $\mathbb{Z} / 2$-trivial Bott manifolds and let $\psi: H^{*}(M(A) ; \mathbb{Z}) \rightarrow H^{*}(M(B) ; \mathbb{Z})$ be any graded ring isomorphism. Since $M(A)$ and $M(B)$ are $\mathbb{Z} / 2-$ trivial, $\alpha_{j}^{A} \equiv 0(\bmod 2)$ and $\alpha_{j}^{B} \equiv 0(\bmod 2)$ for any $j$ by Lemma 6.4. This together with Lemma 4.2 shows that the assumption of Proposition 6.3 is satisfied for the $\psi$, so the theorem follows from Proposition 6.3. 


\section{Cohomology Bott manifolds}

In this section we observe that the invariance of Pontrjagin classes under cohomology ring isomorphisms holds for a slightly wider class of manifolds than the class of Bott manifolds.

As mentioned in the introduction, Bott manifolds are examples of toric manifolds, where a toric manifold of complex dimension $n$ is a smooth compact algebraic variety of complex dimension $n$ with an algebraic action of $\left(\mathbb{C}^{*}\right)^{n}$ having an open dense orbit. Several topological analogs of toric manifolds has been studied and the widest class among them is the class of torus manifolds. A torus manifold is a $2 n$-dimensional closed smooth manifold $M$ with an effective smooth action of an $n$-dimensional torus $T=\left(S^{1}\right)^{n}$ having a fixed point. A toric manifold with the restricted action of $T$ is a torus manifold. We remark that a torus manifold is not necessarily simply connected while any toric manifold is simply connected.

We review some general facts on torus manifolds from [12; 13]. Suppose that the cohomology ring of a torus manifold $M$ of real dimension $2 n$ is generated by degreetwo elements. Then the orbit space $Q:=M / T$ is a nice manifold with corners, any face is acyclic and any multiple intersection of faces in $Q$ is connected unless the intersection is empty, so $Q$ looks like a simple polytope. Let $p: M \rightarrow Q$ be the quotient map and $Q_{i}(i=1,2, \ldots, m)$ be the facets of $Q$. Then $M_{i}:=p^{-1}\left(Q_{i}\right)$ is a closed smooth codimension-two submanifold of $M$ and fixed pointwise under some circle subgroup of $T$. We choose orientations on $M$ and the $M_{i}$ and let $x_{i} \in H^{2}(M ; \mathbb{Z})$ be the Poincaré dual to the cycle $M_{i}$ for $i=1,2, \ldots, m$. Then

$$
p(M)=\prod_{i=1}^{m}\left(1+x_{i}^{2}\right) .
$$

A primitive element $v_{i} \in H_{2}(B T ; \mathbb{Z})$ is uniquely associated to each $M_{i}$. In fact, through a natural identification of $H_{2}(B T ; \mathbb{Z})$ and the group of homomorphisms from $S^{1}$ to $T, v_{i}\left(S^{1}\right)$ is the circle subgroup which fixes $M_{i}$ pointwise. There are two such primitive elements and the orientations on $M$ and $M_{i}$ determine $v_{i}$ uniquely. These $v_{i}$ appear in the presentation of the cohomology ring of $M$ as

$$
H^{*}(M ; \mathbb{Z})=\mathbb{Z}\left[x_{1}, \ldots, x_{m}\right] / \mathcal{K},
$$

where $\mathcal{K}$ is the ideal generated by the two types of elements

(1) $\prod_{i \in I} x_{i}$ for $I \subset\{1,2, \ldots, m\}$ such that $\bigcap_{i \in I} Q_{i}=\varnothing$,

(2) $\sum_{i=1}^{m}\left\langle u, v_{i}\right\rangle x_{i}$ for $u \in H^{2}(B T ; \mathbb{Z})$, 
where $\langle\cdot, \cdot\rangle$ denotes the natural pairing between $H^{2}(B T ; \mathbb{Z})$ and $H_{2}(B T ; \mathbb{Z})$. The vectors $v_{i}$ satisfy the following nonsingularity condition $(*)$ :

For any subset $I$ of $\{1,2, \ldots, m\}$ with cardinality $n$ such that (*) $\bigcap_{i \in I} Q_{i} \neq \varnothing$ (in fact, $\bigcap_{i \in I} Q_{i}$ is a vertex), the set $\left\{v_{i} \mid i \in I\right\}$ forms a basis of $H_{2}(B T ; \mathbb{Z})$.

We say that a closed smooth manifold is a cohomology Bott manifold if its integral cohomology ring is isomorphic to that of some Bott manifold $B_{n}$ as graded rings. Assume that a torus manifold $M$ is a cohomology Bott manifold. The following proposition shows that the Pontrjagin class of $M$ is also of the same form as that of $B_{n}$, so the invariance of Pontrjagin classes under cohomology ring isomorphisms holds for the class of cohomology Bott manifolds.

Proposition 7.1 Suppose that a torus manifold $M$ of real dimension $2 n$ is a cohomology Bott manifold. Then there are elements $x_{1}, x_{2}, \ldots, x_{n} \in H^{2}(M ; \mathbb{Z})$ such that

$$
H^{*}(M ; \mathbb{Z})=\mathbb{Z}\left[x_{1}, x_{2}, \ldots, x_{n}\right] /\left(x_{j}^{2}-\alpha_{j} x_{j} \mid j=1,2, \ldots, n\right),
$$

where $\alpha_{1}=0$ and $\alpha_{j}$ for $j=2, \ldots, n$ is a linear combination of $x_{1}, \ldots, x_{j-1}$ over $\mathbb{Z}$ and that $p(M)=\prod_{i=1}^{n}\left(1+\alpha_{j}^{2}\right)$.

Proof We will freely use the notation introduced above. If $M$ is a genuine Bott manifold, then it is known and easy to see that the orbit space $Q$ is an $n$-cube. As for a cohomology Bott manifold $M$, the orbit space $Q$ is not necessarily an $n$-cube but the argument in [6, Theorem 5.3] or [14, Theorem 5.5] shows that the face poset of $Q$ is isomorphic to that of an $n$-cube. Therefore, $Q$ has $2 n$ facets (ie, $m=2 n$ ) and one can order the facets $Q_{i}$ of $Q$ in such a way that $Q_{j} \cap Q_{j+n}=\varnothing$ for $j=1,2, \ldots, n$. Then (1) after (7-2) is nothing but $x_{j} x_{n+j}$ for $j=1,2, \ldots, n$. Since $\bigcap_{j=1}^{n} Q_{n+j}$ is a vertex, the set $\left\{v_{n+1}, v_{n+2}, \ldots, v_{2 n}\right\}$ forms a basis of $H_{2}(B T ; \mathbb{Z})$ by the nonsingularity condition $(*)$ above. Let $\left\{u_{1}, \ldots, u_{n}\right\}$ be the basis of $H^{2}(B T ; \mathbb{Z})$ such that $\left\langle u_{i}, v_{n+j}\right\rangle=\delta_{i j}$, where $\delta_{i j}$ denotes Kronecker delta. Taking $u=u_{j}$ in (2) after (7-2), one has $x_{n+j}=-\sum_{i=1}^{n}\left\langle u_{j}, v_{i}\right\rangle x_{i}$ in $H^{*}(M ; \mathbb{Z})$; so (7-2) reduces to

$$
H^{*}(M ; \mathbb{Z})=\mathbb{Z}\left[x_{1}, x_{2}, \ldots, x_{n}\right] /\left(x_{j}\left(\sum_{i=1}^{n}\left\langle u_{j}, v_{i}\right\rangle x_{i}\right) \mid j=1,2, \ldots, n\right) .
$$

The nonsingularity condition ( $*$ ) above implies that every principal minor of an $n \times n$ matrix $\Lambda$ with $\left\langle u_{j}, v_{i}\right\rangle$ as $(i, j)^{\text {th }}$ entry is \pm 1 . We may assume that all the diagonal entries of the matrix $\Lambda$ are -1 , by changing the orientations on $M_{i}$ for $i=1, \ldots, n$ if necessary. Then it is shown in [7] that the matrix $\Lambda$ is conjugate to an upper-triangular matrix by a permutation matrix, where we again use the assumption that $M$ is a 
cohomology Bott manifold. Since conjugation by a permutation matrix is nothing but a re-ordering of facets, we may assume that $\Lambda$ is upper-triangular and its diagonal entries are all -1 . This shows that $x_{n+j}=x_{j}-\alpha_{j}$, where $\alpha_{1}=0$ and $\alpha_{j}$ for $j=2, \ldots, n$ is a linear combination of $x_{1}, \ldots, x_{j-1}$ over $\mathbb{Z}$ and (7-4) reduces to (7-3).

Finally, since $x_{n+j}=x_{j}-\alpha_{j}$ and $x_{j}\left(x_{j}-\alpha_{j}\right)=0$ in $H^{*}(M ; \mathbb{Z})$ for $j=1,2, \ldots, n$, it follows from (7-1) that

$$
\begin{aligned}
p(M) & =\prod_{j=1}^{n}\left(1+x_{j}^{2}\right)\left(1+x_{n+j}^{2}\right)=\prod_{j=1}^{n}\left(1+x_{j}^{2}\right)\left(1+\left(x_{j}-\alpha_{j}\right)^{2}\right) \\
& \left.=\prod_{j=1}^{n}\left(1+x_{j}^{2}+\left(x_{j}-\alpha_{j}\right)^{2}\right)\right)=\prod_{j=1}^{n}\left(1+2 x_{j}\left(x_{j}-\alpha_{j}\right)+\alpha_{j}^{2}\right)=\prod_{j=1}^{n}\left(1+\alpha_{j}^{2}\right) .
\end{aligned}
$$

This completes the proof of the proposition.

Corollary 7.2 Let $M$ be a torus manifold and $B_{n}$ a Bott manifold. Then, any graded ring isomorphism $\varphi: H^{*}(M ; \mathbb{Z}) \rightarrow H^{*}\left(B_{n} ; \mathbb{Z}\right)$ preserves their Pontrjagin classes.

Remark 7.3 We remark that if a torus manifold $M$ in Proposition 7.1 is simply connected, then $M$ is indeed homeomorphic to a Bott manifold. This follows from the proof of Proposition 7.1 together with [19, Theorem 3.4].

\section{Concluding remarks}

We conclude this paper with some remarks on the automorphisms of the cohomology ring of a Bott manifold $B_{n}$. Proposition 4.1 and Lemma 4.2 say that, given a graded ring automorphism $\psi$ of $H^{*}\left(B_{n} ; \mathbb{Z}\right)$, there is a permutation $\sigma$ on $[n]$ and a $q_{j} \in\left\{ \pm \frac{1}{2}, \pm 1, \pm 2\right\}$ such that $\psi\left(y_{j}\right)=q_{j} y_{\sigma(j)}$ for each $j=1,2, \ldots, n$. Therefore, assigning $\left(q_{j} /\left|q_{j}\right|\right)_{j=1}^{n}$, together with $\sigma$, to $\psi$, we obtain a monomorphism

$$
\operatorname{Aut}\left(H^{*}\left(B_{n} ; \mathbb{Z}\right)\right) \hookrightarrow\{ \pm 1\}^{n} \rtimes \mathfrak{S}_{n} .
$$

Here $\operatorname{Aut}\left(H^{*}\left(B_{n} ; \mathbb{Z}\right)\right)$ denotes the group of graded ring automorphisms of $H^{*}\left(B_{n} ; \mathbb{Z}\right)$ and $\{ \pm 1\}^{n} \rtimes \mathfrak{S}_{n}$ is the signed permutation group on $[n]$, that is, the semidirect product of the $n$-fold product $\{ \pm 1\}^{n}$ of the order-two group $\{ \pm 1\}$ and the permutation group $\mathfrak{S}_{n}$ on $[n]$, where the action of $\mathfrak{S}_{n}$ on $\{ \pm 1\}^{n}$ is the natural permutation of factors of $\{ \pm 1\}^{n}$. Lemma 4.2 in [10] implies that any automorphism in the subgroup $\{ \pm 1\}^{n}$ of $\{ \pm 1\}^{n} \rtimes \mathfrak{S}_{n}$ can be realized by a diffeomorphism of $B_{n}$ and our Lemma 6.1 implies that some elements of $\mathfrak{S}_{n}$ can also be realized by diffeomorphisms of $B_{n}$. However, any $q_{j}$ is equal to \pm 1 for the cohomology automorphisms induced by those diffeomorphisms, 
so if some $q_{j}$ is not \pm 1 then our results do not prove that the cohomology automorphism is induced by a diffeomorphism. This is the reason why we needed to assume the $\mathbb{Z} / 2$-triviality in Theorem 1.3.

As remarked in the introduction, the Hirzebruch surface $\mathbb{C P}^{2} \# \overline{\mathbb{C P}^{2}}$ is not $\mathbb{Z} / 2$-trivial (although it is $\mathbb{Q}$-trivial). If we put $B_{2}=\mathbb{C P}^{2} \# \overline{\mathbb{C P}^{2}}$, then

$$
H^{*}\left(B_{2} ; \mathbb{Z}\right)=\mathbb{Z}\left[x_{1}, x_{2}\right] /\left(x_{1}^{2}, x_{2}^{2}-x_{1} x_{2}\right),
$$

so $y_{1}=x_{1}$ and $y_{2}=x_{2}-\frac{1}{2} x_{1}$. The map (8-1) above is an isomorphism in this case. In fact, $\operatorname{Aut}\left(H^{*}\left(B_{2} ; \mathbb{Z}\right)\right)$ is generated as a group by the following three automorphisms:

(1) $\left(x_{1}, x_{2}\right) \rightarrow\left(-x_{1},-x_{1}+x_{2}\right)$, so $\left(y_{1}, y_{2}\right) \rightarrow\left(-y_{1}, y_{2}\right)$,

(2) $\left(x_{1}, x_{2}\right) \rightarrow\left(x_{1}, x_{1}-x_{2}\right)$, so $\left(y_{1}, y_{2}\right) \rightarrow\left(y_{1},-y_{2}\right)$,

(3) $\left(x_{1}, x_{2}\right) \rightarrow\left(-x_{1}+2 x_{2}, x_{2}\right)$, so $\left(y_{1}, y_{2}\right) \rightarrow\left(2 y_{2}, \frac{1}{2} y_{1}\right)$.

The automorphisms (1) and (2) generate the subgroup $\{ \pm 1\}^{2}$ while the automorphism (3) generates the subgroup $\mathfrak{S}_{2}$. As remarked above, the automorphisms (1) and (2) are induced by diffeomorphisms of $B_{2}$. One can see that the automorphism (3) is also induced by a diffeomorphism of $B_{2}$. In fact, the diffeomorphism of type 2 in the proof of [3, Lemma 5.2] induces the automorphism (3) up to sign. For the convenience of the reader, we shall review the construction of this diffeomorphism. We take involutions

$$
s:\left[z_{1}, z_{2}, z_{3}\right] \rightarrow\left[\bar{z}_{1}, \bar{z}_{2}, \bar{z}_{3}\right] \text { on } \mathbb{C P}^{2}, \quad t:\left[z_{1}, z_{2}, z_{3}\right] \rightarrow\left[z_{1}, z_{2},-z_{3}\right] \text { on } \overline{\mathbb{C P}^{2}}
$$

where $\left[z_{1}, z_{2}, z_{3}\right]$ denotes the homogeneous coordinate of $\mathbb{C P}^{2}$ and $\bar{z}$ denotes the conjugate of a complex number $z$. The fixed point set of $s$ is $\mathbb{R P}^{2}$ while that of $t$ consists of a point and $\mathbb{C P}^{1}$. Choose a point from each of $\mathbb{R P}^{2}$ and $\mathbb{C P}^{1}$ respectively and take the equivariant connected sum of $\mathbb{C P}^{2}$ and $\overline{\mathbb{C P}^{2}}$ around the chosen fixed points. Then the resulting involution on $B_{2}=\mathbb{C P}^{2} \# \overline{\mathbb{C P}^{2}}$ is the desired one (see the proof of [3, Lemma 5.2] for more details). This construction heavily depends on the explicit description of $\mathbb{C P}^{2} \# \overline{\mathbb{C P}^{2}}$ and we do not know how to find such a diffeomorphism for a non- $\mathbb{Z} / 2$-trivial Bott manifold $B_{n}$, although such a diffeomorphism can be found when $B_{n}$ is $\mathbb{Q}$-trivial [3]. If we overcome this difficulty, then we could solve the strong cohomological rigidity conjecture for Bott manifolds completely.

Acknowledgements The second and third authors would like to thank Professor Junzo Watanabe for stimulating discussions. The first author was partially supported by Basic Science Research Program through the National Research Foundation of Korea (NRF) funded by the Ministry of Education (NRF-2011-0024975) and the TJ Park Science Fellowship funded by the POSCO TJ Park Foundation. The second author was partially supported by JSPS KAKENHI 25400095. The third author was partially supported by JSPS KAKENHI 25400043. 


\section{References}

[1] A Borel, F Hirzebruch, Characteristic classes and homogeneous spaces, I, Amer. J. Math. 80 (1958) 458-538 MR0102800

[2] S Choi, Classification of Bott manifolds up to dimension eight arXiv:1112.2321 to appear in Proc. Edinburgh Math. Soc.

[3] S Choi, M Masuda, Classification of $\mathbb{Q}$-trivial Bott manifolds, J. Symplectic Geom. 10 (2012) 447-461 MR2983437

[4] S Choi, M Masuda, D Y Suh, Topological classification of generalized Bott towers, Trans. Amer. Math. Soc. 362 (2010) 1097-1112 MR2551516

[5] S Choi, M Masuda, D Y Suh, Rigidity problems in toric topology: A survey, Tr. Mat. Inst. Steklova 275 (2011) 188-201 MR2962979 In Russian; translated in Proc. Stelov Inst. Math. 275 (2011) 177-190

[6] S Choi, T E Panov, D Y Suh, Toric cohomological rigidity of simple convex polytopes, J. Lond. Math. Soc. 82 (2010) 343-360 MR2725043

[7] S Choi, D Y Suh, Properties of Bott manifolds and cohomological rigidity, Algebr. Geom. Topol. 11 (2011) 1053-1076 MR2792373

[8] Y Civan, N Ray, Homotopy decompositions and K-theory of Bott towers, K-Theory 34 (2005) 1-33 MR2162899

[9] D Crowley, M Kreck, Hirzebruch surfaces, Bulletin of the Manifold Atlas (2011)

[10] H Ishida, Filtered cohomological rigidity of Bott towers, Osaka J. Math. 49 (2012) 515-522 MR2945760

[11] Manifold atlas project, Petrie conjecture, electronic resource Available at http:// www .map.mpim-bonn.mpg.de/Petrie_conjecture

[12] M Masuda, Unitary toric manifolds, multi-fans and equivariant index, Tohoku Math. J. 51 (1999) 237-265 MR1689995

[13] M Masuda, T E Panov, On the cohomology of torus manifolds, Osaka J. Math. 43 (2006) 711-746 MR2283418

[14] M Masuda, T E Panov, Semi-free circle actions, Bott towers, and quasitoric manifolds, Mat. Sb. 199 (2008) 95-122 MR2452268 In Russian; translated in Sb. Math. 199 (2008) 1201-1223

[15] M Masuda, D Y Suh, Classification problems of toric manifolds via topology, from: "Toric topology", (M Harada, Y Karshon, M Masuda, T E Panov, editors), Contemp. Math. 460, Amer. Math. Soc. (2008) 273-286 MR2428362

[16] T Petrie, Smooth $S^{1}$ actions on homotopy complex projective spaces and related topics, Bull. Amer. Math. Soc. 78 (1972) 105-153 MR0296970

[17] T Petrie, Torus actions on homotopy complex projective spaces, Invent. Math. 20 (1973) 139-146 MR0322893 
[18] D Sullivan, Infinitesimal computations in topology, Inst. Hautes Études Sci. Publ. Math. (1977) 269-331 MR0646078

[19] M Wiemeler, Torus manifolds and non-negative curvature arXiv:1401.0403

Department of Mathematics, Ajou University

San 5, Woncheon-dong, Yeongtong-gu, Suwon 443-749, South Korea

Department of Mathematics, Osaka City University

3-3-138, Sugimoto, Sumiyoshi-ku, Osaka-shi 558-8585, Japan

Department of Pure and Applied Mathematics

Graduate School of Information Science and Technology

Osaka University, Toyonaka, Osaka 560-0043, Japan

schoi@ajou.ac.kr, masuda@sci.osaka-cu.ac.jp, s-murai@ist.osaka-u.ac.jp

Received: 6 May 2014 Revised: 15 September 2014 